\title{
A bag of tricks for automated de novo design of molecules with the desired properties: application to EGFR inhibitor discovery
}

\author{
Maria Korshunoval, ${ }^{1, *}$, Niles Huang ${ }^{3}$, Stephen Capuzzi ${ }^{4}$, Dmytro S. Radchenko ${ }^{5,6}$, Olena Savych ${ }^{5}$, \\ Yuriy S. Moroz ${ }^{6,7}$, Carrow I. Wells, ${ }^{8}$ Timothy M. Willson ${ }^{8}$, \\ Alexander Tropsha ${ }^{4}$, Olexandr Isayev ${ }^{1,2^{*}}$ \\ ${ }^{1}$ Department of Chemistry, Mellon College of Science, Carnegie Mellon University, Pittsburgh, \\ Pennsylvania \\ ${ }^{2}$ Computational Biology Department, School of Computer Science, Carnegie Mellon University, \\ Pittsburgh, Pennsylvania \\ ${ }^{3}$ Department of Biochemistry, University of Oxford, Oxford, United Kingdom \\ ${ }^{4}$ Laboratory for Molecular Modeling, UNC Eshelman School of Pharmacy, University of North Carolina \\ at Chapel Hill, Chapel Hill, North Carolina \\ ${ }^{5}$ Enamine Ltd, 78 Chervonotkatska Street, 02094, Ukraine \\ ${ }^{6}$ Taras Shevchenko National University of Kyiv, Volodymyrska Street 60, Kyiv 01601, Ukraine \\ ${ }^{7}$ Chemspace LLC, Chervonotkatska Street 85, Suite 1, Kyiv 02094, Ukraine \\ ${ }^{8}$ Structual Genomics Consortium, UNC Eshelman School of Pharmacy, University of North Carolina at \\ Chapel Hill, Chapel Hill, North Carolina \\ *Correspondence: mariewelt@cmu.edu (M.K.); olexandr@olexandrisayev.com (O.I.)
}

\begin{abstract}
Deep generative neural networks have been used increasingly in computational chemistry for de novo design of molecules with desired properties. Many deep learning approaches employ reinforcement learning for optimizing the target properties of the generated molecules. However, the success of this approach is often hampered by the problem of sparse rewards as the majority of the generated molecules are expectedly predicted as inactives. We propose several technical innovations to address this problem and improve the balance between exploration and exploitation modes in reinforcement learning. In a proof-of-concept study, we demonstrate the application of the deep generative recurrent neural network enhanced by several novel technical tricks to designing experimentally validated potent inhibitors of the epidermal growth factor (EGFR). The proposed technical solutions are expected to substantially improve the success rate of finding novel bioactive compounds for specific biological targets using generative and reinforcement learning approaches.
\end{abstract}


Deep and reinforcement learning in drug discovery. The development and application of deep generative models for de novo design of molecules with the desired properties have emerged as an important modern research direction in Computer-Assisted Drug Discovery (CADD). ${ }^{1-4}$ Deep generative models can be classified by the types of molecular representation employed in model development. The most commonly used types are SMILES strings ${ }^{5}$ and molecular graphs. Multiple models for generating SMILES strings ${ }^{6-9}$ and molecular graphs ${ }^{10-14}$ corresponding to synthetically feasible novel molecues have been proposed. Many of such models use reinforcement learning $(\mathrm{RL})^{7,15,16}$ techniques for optimizing properties of the generated molecules. For example, Olivecrona et al. ${ }^{6}$ and Blaschke et al. ${ }^{17}$ proposed the REINVENT algorithm and memory-assisted reinforcement learning, respectively, and demonstrated how these approaches could maximize the predicted activity of generated molecules against the 5hydroxytryptamine receptor type 1A (HTR1A) and the dopamine type 2 receptor (DRD2). Another recent example is the RationaleRL algorithm proposed by Jin et al. ${ }^{18}$ The authors used RationaleRL to maximize the predicted activity of inhibitors against glycogen synthase kinase-3 beta (GSK3 $\beta$ ) and c-Jun N-terminal kinase-3 (JNK3). Unfortunately, the aforementioned studies included no experimental validation of the proposed computational hits. Notably, Zhavoronkov et a ${ }^{19}$ not only proposed a novel generative tensorial reinforcement learning algorithm, but also used their method to design potent DDR1 kinase inhibitors, and performed experimental validation of virtual hits.

Most theoretical works on de novo molecular design employ a series of benchmark tasks such as maximization of properties that can be assessed for every molecule, such as $\log \mathrm{P}^{20}$, $\mathrm{QED}^{21}$, or the benchmark collection proposed in GuacaMol. ${ }^{22}$ Such tasks employ objective metrics obtained directly from a molecule's SMILES ${ }^{5}$ or underlying molecular graph through a scoring function. These scoring functions return continuous values that can be used to assign a reward to generated molecules. For example, the Quantitative Estimate of Druglikeness score (QED) has values between 0 and 1.0, with 0 being least drug-like and 1.0 being most drug-like. In such a case, every generated molecule would receive a continuous score: the bigger score values will correspond to bigger reward values, and vice versa. Moreover, a naïve generative model pretrained on a dataset of drug-like compounds such as $\mathrm{ChEMBL}^{23}$ would produce molecules with relatively high QED values. In this case, optimization of the generative model via reinforcement learning will proceed efficiently as every generated molecule would get a score. Indeed, the efficient optimization of the QED score has been demonstrated many times in the literature. ${ }^{10,20,24}$

Problem of sparse rewards in reinforcement learning. In contrast to physical properties such as $\log \mathrm{P}$ that can be calculated directly from molecular structure, the biological activity of a novel compound designed to bind the desired protein target cannot be predicted from its chemical structure alone. A common way to predict the binding affinity of novel, untested ligands is by using Quantitative Structure-Activity Relationship (QSAR) models ${ }^{25,26}$ trained on historical experimental data for a protein target of interest using machine learning techniques. These models have either continuous outputs ( $\mathrm{pKd}$, pIC50, etc.) for regression problems or binary outputs (active/inactive class label) for classification problems. QSAR models could, in principle, be used to construct a reward function for reinforcement learning to optimize the binding affinity of 
generated molecules, as was shown, for instance, in our previous publication. ${ }^{7}$ However, unlike physical molecular properties like LogP that every molecule possesses, specific bioactivity is a target property that exists for only a small fraction of molecules, which leads to the reward sparseness in the generative models. This sparse rewards problem represents a serious obstacle for the effective use of reinforcement learning for designing molecules with high activity. Indeed, the low success probability often leads to the overwhelming majority of training trajectories resulting in a zero reward, which implies that the reinforcement learning agent or policy network struggles to explore the environment and learn the optimal strategy for maximizing the expected reward. Thus, a promising molecule with high bioactivity for a protein of interest is unlikely to be observed if molecules are randomly sampled from a naïve generative model.

Training the generative network to optimize the potency of generated molecules against a desired protein target is an excellent example of a reinforcement learning problem with sparse rewards. In this study, we demonstrate that the naïve generative model produces molecules predicted to be inactive in most cases. Under such a scenario, the naïve generative model rarely observes good examples and fails to maximize the binding affinity of generated ligands. We further address this problem by proposing a set of heuristic approaches (a "bag of tricks") combined with reinforcement learning in the sparse rewards situation to increase the efficiently of optimizing the structures of generated molecules to have higher biological activity. Using the epidermal growth factor receptor (EGFR) ligands as a case study, we show that by combining a reinforcement learning pipeline for generative model optimization with proposed heuristics, we could overcome sparse reward issues and successfully rediscover known active scaffolds for EGFR using the feedback from the classification QSAR model only. In addition to methodological advances, we also performed experimental bioassay validation of the novel generated hit molecules, which confirmed the experimental activity of virtual hits.

Major findings. We performed a series of experiments that resulted in the following chief observations:

1. The generative model trained with only the policy gradient algorithm could not discover any active molecules for EGFR due to sparse rewards.

2. The combination of policy gradient algorithm with proposed fine-tuning by (i) transfer learning, (ii) experience replay, and (iii) real-time reward shaping resulted in much better exploration and an increased number of generated molecules with high active class probabilities.

3. Experimental testing of selected computational hits that could be obtained from a commercial source validated the efficiency of our novel approach for discovering novel bioactive molecules.

Below, we discuss how we arrived at the above observations. Overall, the section consists of two main parts. In the first part, we describe our computational analysis concerning the first two observations. In the second part, we discuss the generation, selection, and experimental bioactivity testing of computational hit compounds for an important cancer biological target, epidermal growth factor receptor (EGFR). The most active compound featured a privileged EGFR scaffold found in the known active molecules. Notably, the training set was not enriched for this scaffold 
as compared to other scaffolds and this scaffold was not used selectively as part of the reinforcement learning procedure.

Model pipeline. Neural network training is a nontrivial task as its hyperparameter values define a training protocol. Due to the high number of hyperparameters, the training hyperparameter space is vast. To complicate things further, neural network training is a computationally expensive task that can last hours to days. The choice of training hyperparameters thus has a significant influence on model quality. We sought to run a benchmark experiment to investigate how different training techniques interact and how they affect model quality. As a case study, we performed the optimization of the generative model with reinforcement learning to maximize the predicted probability of active class for EGFR protein. The experimental training pipeline is shown in Figure 1 .

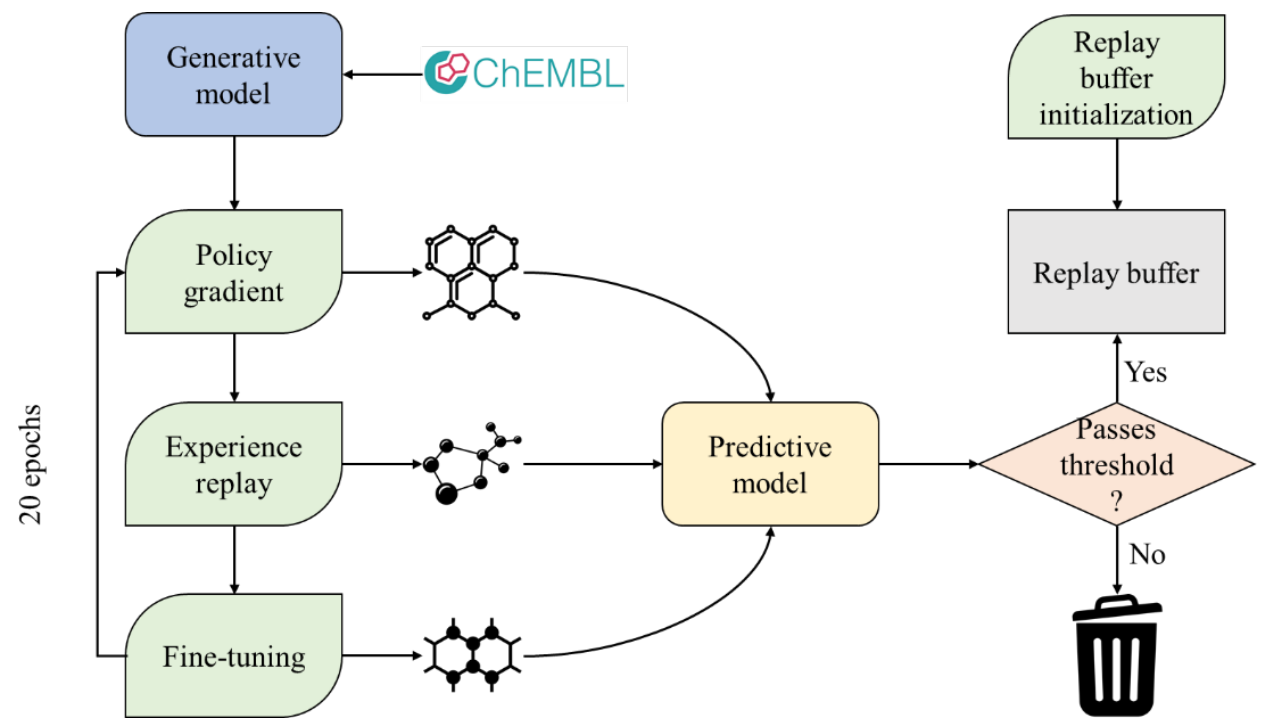

Figure 1: Pipeline of model training. The model was pre-trained on ChEMBL data and then trained for 20 epochs. Each epoch consists of three steps: policy gradient, policy experience replay, and fine-tuning. At the end of each step, 3,200 molecules are generated, and molecules with predicted activity exceeding the probability threshold are admitted into the replay buffer. The replay buffer, in turn, influences training at the policy replay and fine-tuning steps. At the end of the training, the model generates 16,000 molecules for evaluation. We modified the number of iterations for all 3 steps in each epoch to understand their effects on training. We also used different libraries to initialize the replay buffer to understand how the replay buffer can influence model behavior.

As described above, we used the pre-trained ChEMBL model and populated the experience replay buffer with generated active molecules to initialize training. The model was trained using different combinations of policy gradient, experience replay, and fine-tuning. At the end of each substep, 3,200 molecules were generated for intermediate evaluation. If experience replay and/or fine-tuning were used, molecules with predicted activity exceeding the probability threshold were admitted into the experience replay buffer. In turn, the replay buffer influences training at the policy replay and fine-tuning steps in the next epoch if used. At the end of the training, the model generated 16,000 molecules for evaluation. We first trained the model for a variable number of epochs and verified that the model learns significantly after 20 epochs (Figure S1). 
Effect of fine-tuning vs. reinforcement learning. The bar chart shown in Figure 2 summarizes the findings for four representative conditions: 1) policy gradient only, 2) policy gradient and fine-tuning, 3) policy gradient and experience replay, and 4) policy gradient, experience replay, and fine-tuning. We assessed the extent of overfitting by recording the fraction of the generated trajectories that generate valid SMILES strings, which is defined as the ratio of valid and unique SMILES strings over the total number of the generated trajectories. We assessed the extent of model learning by recording the fraction of the generated trajectories resulting in active chemical structures, which is defined as the ratio of valid SMILES strings with predicted EGFR activity (with the arbitrary probability threshold of 0.75 ) over the number of valid and unique SMILES strings generated. Training without replay tricks has a near-zero "active" fraction and the highest "valid" fraction. This observation is consistent with the sparse rewards hypothesis. In the absence of rewards from active molecules, this model effectively trains on the classifier objective. Instead of learning to generate active molecules, the model optimizes valid fraction. Training with a single trick (fine-tuning or experience replay) teaches the model to generate active molecules, albeit at the expense of a lower valid fraction. Training with only fine-tuning results in a lower fraction of valid molecules. Training with both experience replay and fine-tuning yields the best results, with both high active fraction and high valid fraction. A more detailed summary with nine different training conditions is shown in Figure S2.

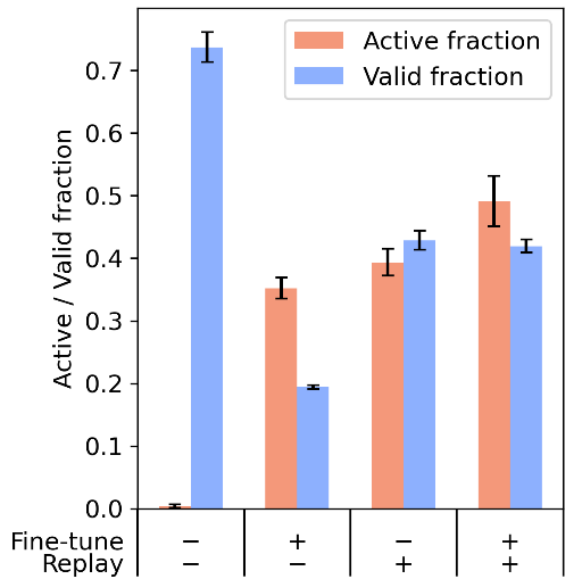

Figure 2: Combined effects of fine-tuning and reinforcement learning. Four conditions are shown here, representing the four combinations of fine-tuning and experience replay. From left to right, the conditions are: 1) no experience replay and no fine-tuning, 2) fine-tuning only, 3) experience replay only, and 4) both experience replay and fine-tuning. Models were trained for 20 epochs. Conditions with fine-tuning used 20 iterations of fine-tuning; those without used 0 iterations of fine-tuning. All training epochs had 25 policy steps, with different ratios of experience replay and policy gradient. Conditions with experience replay used 10 iterations of experience replay and 15 iterations of policy gradient; those without used 0 iterations of experience replay and 25 iterations of policy gradient.

Next, we analyzed the effect of fine-tuning steps on mode collapse. ${ }^{27}$ Mode collapse poses a significant challenge in generative models. Reinforcement learning teaches generative models to produce output with high reward; however, it does not consider the distribution of generated output. Thus, the model can discover a pathological local minimum in the objective function by 
converging to generate a few instances with high reward; in such cases, the model undergoes mode collapse. Such overfitted models explore limited regions of chemical space and are undesirable for library generation.

Our experiments used the active fraction as a proxy for training progress and the valid fraction as a proxy for mode collapse. Two scenarios can decrease valid fraction: 1) the model generates a larger fraction of invalid SMILES strings (fewer valid SMILES strings), or 2) the model suffers from mode collapse and generates many repeats of the same SMILES string (fewer unique SMILES strings). The first factor is caused by the restricted chemical space of higher activity molecules and is specific to the reward function. The second factor is caused by the nature of training and can be controlled.

Mode collapse effect. To investigate how learning affects mode collapse, we ran several experiments where the generative model was trained with 25 iterations of policy gradient and one of $0,20,50,100,200,500$, or 1,000 iterations of fine-tuning per epoch. We recorded valid fraction and active fraction after each epoch. The resulting trajectories are illustrated in Figure 3. Figure 3(A) shows how active fraction, valid fraction, replay threshold, and average reward change with training for a different number of fine-tuning steps used in training. Figure 3(B) shows the joint trajectories of an active fraction and valid fraction change with training for the different number of fine-tuning steps.

Figure 3 shows that when the model uses no fine-tuning, it fails to produce active molecules and maintains a high valid fraction. When the model uses fine-tuning, it learns to generate active molecules at the expense of a lower valid fraction. All runs with fine-tuning experienced a significant drop in a valid fraction in the first epoch of training. This drop may represent a transient phase when the model cannot generate active molecules and partially overfits to the initial molecules in the replay buffer. The decrease in the valid fraction is more pronounced in models that use more fine-tuning iterations, consistent with this proposal. Models with the fewest finetuning iterations have the lowest active fraction and the lowest valid fraction. Over model training, the active fraction is negatively correlated with the valid fraction, suggesting that the model suffers mode collapse as it learns to generate active molecules. Models with higher fine-tuning iterations have progressively higher active fractions and valid fractions. The model appears to increase valid fraction for the highest numbers tested (500 and 1,000 iterations) as it learns. Although models with higher fine-tuning iterations initially experience a more considerable drop in valid fractions, they eventually have higher valid fractions than models with lower fine-tuning iterations.

Similarly, we analyzed the effect of the different number of experience replay steps. All data is shown in Figures S3 and S4. Similar to the fine-tuning benchmark, the model with no experience replay fails to generate active molecules and maintains a high valid fraction. Inclusion of experience replay results in successful learning with a simultaneous decrease in valid fraction. Unlike the fine-tuning benchmark, however, the number of experience replay steps does not clearly affect model quality. In these experiments, model quality is largely determined by the presence or absence of experience replay steps. 


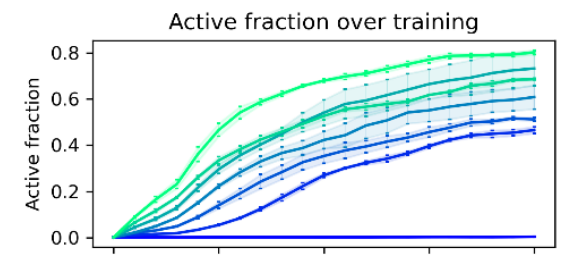

Replay threshold over training

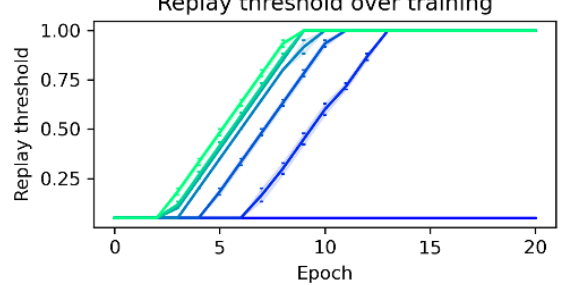

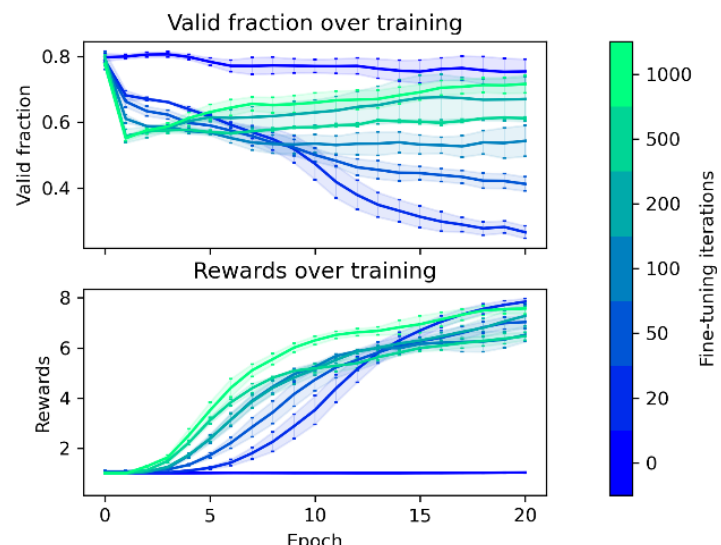

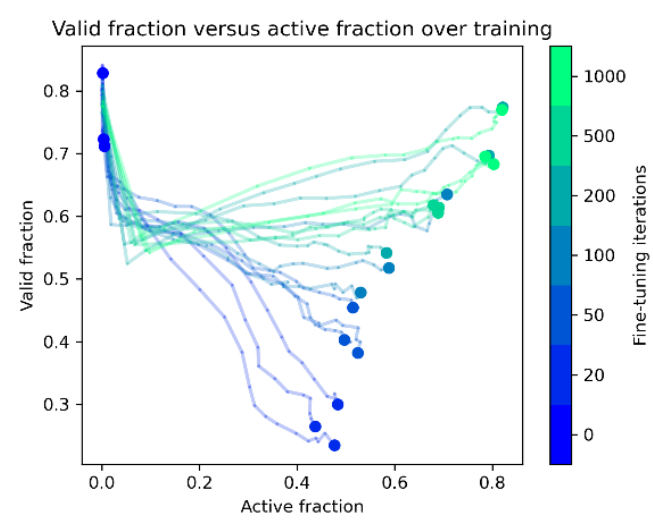

Figure 3: (A) Trajectory of training for different fine-tuning values. Models were trained with 25 iterations of policy gradient and 0,20,50, 100, 200, 500, or 1,000 iterations of fine-tuning per epoch. (B) Evolution of active and valid fractions overtraining. Models were trained with 25 iterations of policy gradient and 0, 20, 50, 100, 200, 500, or 1,000 iterations of fine-tuning per epoch. Solid lines represent training, small dots represent data at each epoch, and large dots represent data from the fully-trained model. Graphs are color-coded by the number of fine-tuning iterations used per epoch.

Experience replay buffer effect. Finally, we investigated different initializations of the experience replay buffer. The experience replay library is typically filled with predicted molecules generated by the model pretrained on the ChEMBL database, but our procedure enables us to use an arbitrary replay library alternatively. Due to sparse rewards, model learning is initially dictated by the replay library. We generated a second replay library with molecules from the Enamine kinase library, which consists of 65,000 small molecules with predicted activity against kinases ${ }^{28}$. This library was chosen based on the expectation that general-purpose kinase inhibitors should contain scaffolds suitable for EGFR kinases.

We first selected molecules with non-zero activities against EGFR, as predicted by the random forest ensemble. We then filtered the active molecules to remove molecules with BemisMurcko scaffolds ${ }^{29}$ present in the historical EGFR data. This step ensured that the replay buffer molecules were dissimilar from known molecules. The final Enamine replay library had 219 molecules (Figure S5). 
This experiment tested three different replay libraries: an empty replay library (Empty buffer), the replay library from the model (Generated actives), and the Enamine library selected as above (Enamine). Figure 4 shows the 12 most common Bemis-Murcko scaffolds ${ }^{29}$ in the generated libraries produced by each of the models. All scaffold calculations were done using the RDKit ${ }^{30}$ package.
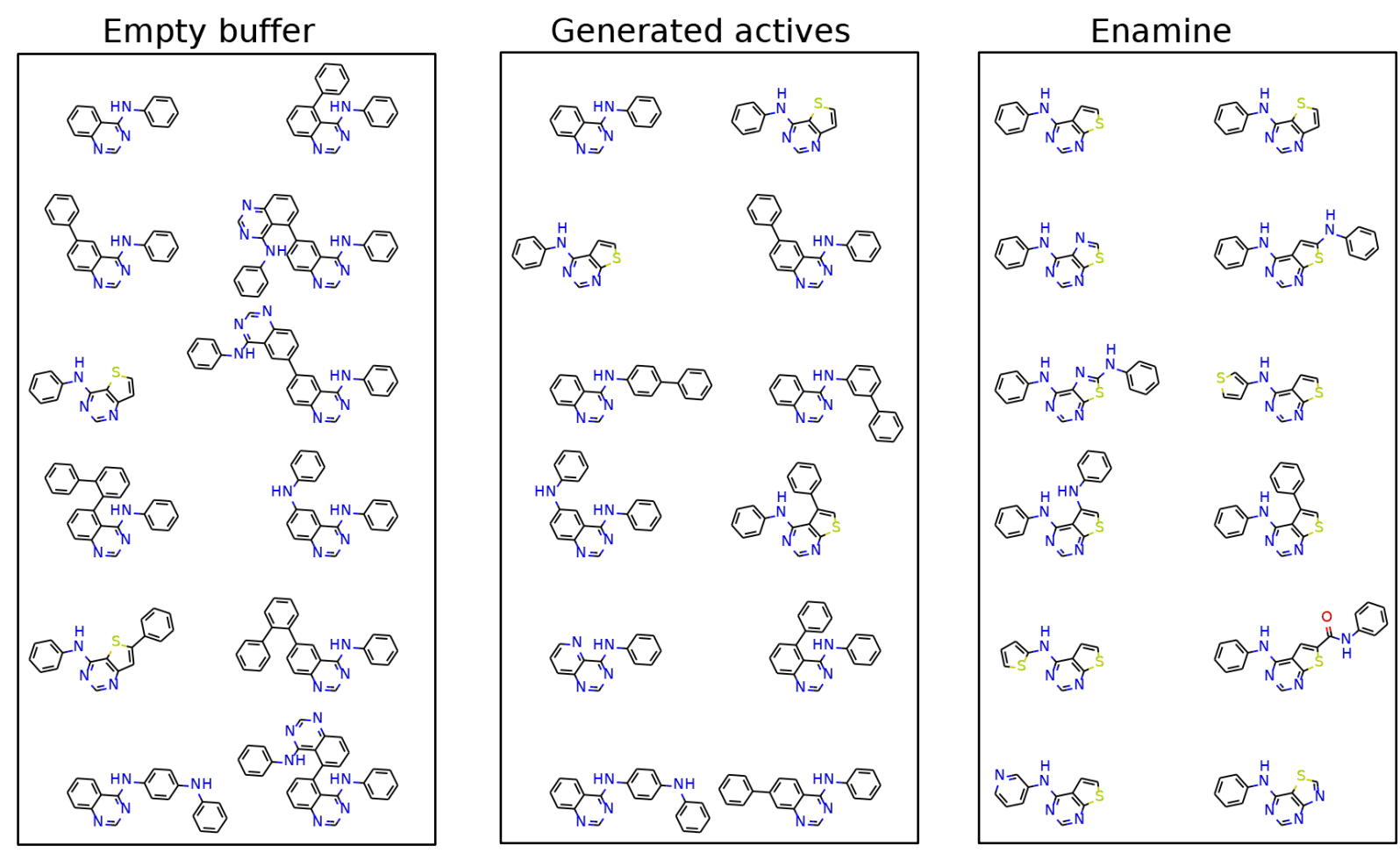

Figure 4: The 12 most common Bemis-Murcko scaffolds for models trained from different libraries. Three replay libraries were tested: an empty replay library (Empty buffer), the replay library from the model (generated actives), and the Enamine library selected as above (Enamine). Models were trained for 20 epochs with 15 iterations of policy gradient, 10 iterations of experience replay, and 20 iterations of fine-tuning per epoch. Scaffolds are sorted with decreasing counts from left to the right, then from top to bottom. The most common scaffolds had counts and percentages as follows: 427 out of 4,077 predicted active molecules (10.5\%) for the empty buffer, 1,232 out of 3,312 (37.2\%) for the generated actives, and 1,763 out of 4,930 (35.8\%) for the Enamine library.

In the generated library produced with replay buffer initialized with compounds from the Enamine kinase library, the main quinazoline scaffold is notably absent. The Enamine-trained library suffers from lower diversity, likely because the initial replay buffer selected from the Enamine kinase library predominantly contains thiophene-fused rings. Such bias was introduced by the predictive model used to select the initial replay buffer, as described in the Methods section. The predictive model favored compounds with thiophene-fused rings. This observation confirms that the initial selection of molecules in the replay library greatly influences the regions of chemical space that the model explores.

The library generated by the Empty buffer-trained model shows clear signs of overfitting, as 3 of the 12 most common scaffolds appear to be duplications of the quinazoline scaffold. The 
first active molecules greatly influence the model admitted into the replay library. When the replay library is initially empty, the model heavily exploits the first active molecules generated. As a result, the empty buffer-trained model explores a very limited region in chemical space (See Figure S6 for similarity distributions).

Generation and selection of hit compounds. With the information obtained through computational analysis, we fixed the model training protocol. We trained the ChEMBL-pretrained model for 20 epochs, with 15 steps of policy gradient, 10 steps of experience replay, and 20 steps of fine-tuning by transfer learning per epoch. Every 2 epochs, we produced snapshot libraries of 16,000 molecules. Each snapshot library included the distribution of active class probability for the generated molecules. Figure 5 illustrates the time-lapse of this distribution. The prominent peaks at 0 and 1 suggest that the model learns by increasing the fraction of highly active molecules, as opposed to generating molecules with progressively higher activities. This observation is likely because the random forest classifiers in the ensemble predictor were trained on the same dataset.

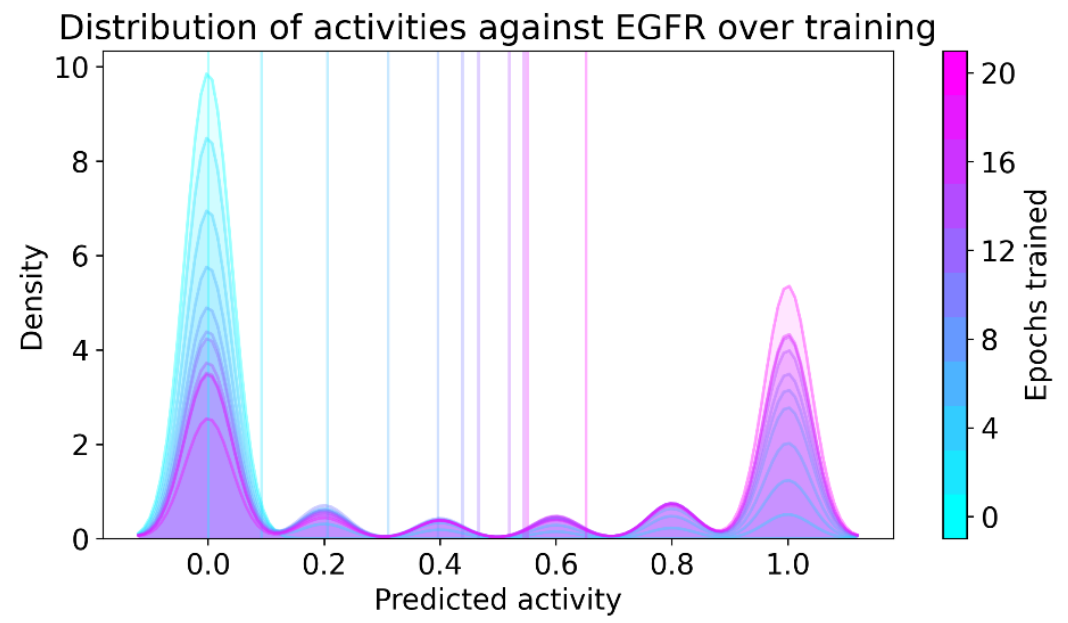

Figure 5: Time-lapse distribution of activity predictions overtraining. Mean predictions are marked by vertical lines. The model was trained for 20 epochs with 15 steps of policy gradient, 10 steps of experience replay, and 20 steps of finetuning per epoch. 1,000 batches of molecules were generated every 2 epochs, and the distribution of predicted activity plotted. Note that the classifier with ensemble size five generates discrete predictions of fifth fractions.

\section{Experimental Validation.}

With few notable exceptions ${ }^{19,31}$, most of the current de novo design publications are purely computational. However, it is important to know how many computationally predicted candidates are experimentally validated by in vitro (at least) assays. For this test, we established the following screening protocol.

The model described in the previous section was used to generate a large library of novel computational hits with high active class probabilities. To enable rapid testing of the computational models all hit molecules were parsed through the Enamine REAL database (Release 2020q1-2, https://enamine.net/library-synthesis/real-compounds/real-database) of $1.36 \mathrm{~B}$ on-demand commercially available molecules. The Enamine REAL (readily accessible) database is based on the synthesis of ultra-large chemical libraries using two- or three-step three-component reaction 
sequences and available starting materials with pre-validated (at least $80 \%$ synthesis success rate) chemical reactivity ${ }^{32}$.

Seventeen computational hit molecules were matched with Enamine REAL. All of the predicted active compounds were derivatives of 4-anilinoquinazoline, a chemotype that was well represented in Enamine REAL (Table S1). The predicted active compounds contained a few small substituents on the quinazoline ring (positions 5-8: $\mathrm{F}, \mathrm{Cl}, \mathrm{Br}, \mathrm{OCH}_{3}$ ) but a wide range of substituents on the 4-anilino group. As a negative control, we selected five molecules predicted to be inactive but containing the same 4-anilinoquinazoline scaffold (Table S1). The twenty three 4anilinoquinazoline analogs were dissolved in DMSO and sent to Reaction Biology (https://www.reactionbiology.com/) for EGFR enzymatic assay screening. Two compounds in the predicted active series were insoluble in DMSO; therefore, biological tests were not performed. The 4-anilinoquinazoline analogs were initially tested in single-dose duplicate mode at a concentration of $1 \mu \mathrm{M}$ and percent inhibition relative to DMSO control was determined (Table $\mathrm{S} 1)$. Staurosporine was used as a reference EGFR tyrosine kinase inhibitor ${ }^{33,34}$.

Table 1. Data for EGFR kinase inhibiton of compounds 1-4.

\begin{tabular}{|c|c|c|c|c|c|}
\hline Compound & Catalog ID & pIC50 & Structure & $\begin{array}{l}\text { Nearest neighbor } \\
\text { (NN) from the } \\
\text { training set }\end{array}$ & $\begin{array}{c}\text { pChEMBL } \\
\text { for } N N\end{array}$ \\
\hline 1 & Z1192045732 & 7.5 & & & 7.6 \\
\hline 2 & Z1576525970 & 7.4 & & & 7.6 \\
\hline 3 & Z1182636554 & 6.7 & & & 7.3 \\
\hline 4 & Z1823625743 & 5.9 & & & 7.3 \\
\hline & & & & $\begin{array}{c}\text { Most active from } \\
\text { ChEMBL }\end{array}$ & $\sim 10$ \\
\hline
\end{tabular}

Four 4-anilinoquinazolines from the predicted hit set showed $>40 \%$ inhibition of EGFR enzyme activity in the $1 \mu \mathrm{M}$ single dose assay (Table S1), while all five of the negative control analogs were inactive. Notably, the four active compounds contained only small substituents $(\mathrm{Br}$, $\mathrm{NH}_{2}, \mathrm{CH}_{3}$ ) at the 4' position of the 4-anilino group (Table S1) paired with halogen substitution on 
the 5,6 , or 8 positions of quinazoline core. Surprisingly, however, the 4'-fluroanilino-6fluroquinazoline analog was not active. Notably, all of the analogs with large linear or branched substituents at the 4' position were inactive in the enzyme assay. The four active compounds from the single-dose assay were further tested in 10-dose $\mathrm{IC}_{50}$ mode with 3-fold serial dilution starting at $10 \mu \mathrm{M}$ to determine their EGFR inhibition potency. The 4-anilinoquinazolines 1 and $\mathbf{2}$ (Table 1) were potent EGFR inhibitors with $\mathrm{IC}_{50}<100 \mathrm{nM}$, comparable to the potency of staurosporine (Table S1). The 4-anilinoquinazolines 3 was slightly less potent with an $\mathrm{IC}_{50}=210 \mathrm{nM}$. The analog 4 was the least potent with $\mathrm{IC}_{50}=1.4 \mu \mathrm{M}$.

Each of the active compounds 1-4 had a 3'-halogen substituted 4-anilinoquinazoline as a close neighbor in the training set that was reported to have a similar EGFR inhibition potency (Table 1). The most potent EGFR inhibitor from ChEMBL was N-(3-bromophenyl)quinazoline4,7-diamine (CHEMBL420624), which had activity at sub-nanomolar concentrations. Although all five out of the negative control compounds were inactive in the EGFR enzyme assay, it should be noted that they each contain large linear or branched substituents at the 4'-position of the aniline. Analogs with the same or similar substitution on the aniline that were selected to be active in the computational model were also shown to be inactive in the EGFR assay (Table S1).

Summary of the study. Herein, we proposed several new improvements to the heuristics used to optimize properties of molecules created by generative neural networks with reinforcement learning and sparse rewards. Sparse rewards are commonly observed when maximizing the bioactivity of generated molecules for a specific target protein. Thus, classic reinforcement learning algorithms such as policy gradient or Q-learning are not sufficient for such tasks. In contrast, our proposed tweaks, i.e., fine-tuning with transfer learning, experience replay, and realtime reward shaping, aim to extract informative feedback from the sparse reward signal and keep a healthy balance between exploration and exploitation. As a result of our study, we came up with a list of crucial points to consider when optimizing generative models with reinforcement learning.

1. We recommend considering the sparsity of the rewards and the desired level of balance between exploration and exploitation when selecting the right strategy for performing optimization in each case. The real-time reward shaping can be helpful in a sparse rewards scenario while unnecessary in cases when the reward feedback is sufficient (such as QED or LogP optimization).

2. The fine-tuning by transfer learning achieves a high level of exploitation, especially when used with known molecules. However, it will unlikely discover any chemotypes beyond the ones used for training.

3. The experience replay requires a rich and diverse pool of experience trajectories. Otherwise, this technique may also result in over-exploitation of replay examples. However, it can be a powerful tool to explore the chemical space and deal with sparse rewards in tandem with a policy gradient.

The optimized protocol was subject to a blind experimental validation. Out of fifteen tested compounds that were predicted active, four were confirmed in an EGFR enzyme assay. Two out of four compounds had nanomolar EGFR inhibition activity comparable to that of staurosporine. The overall hit rate was $\sim 27 \%$. Additionally, five compounds with the same scaffold as in active 
compounds but predicted as inactive were used as a negative control. All five compounds were confirmed as inactive. The obtained hit rate is on par with traditional virtual screening projects where molecule selection is guided by an expert medicinal chemist. However, in this work, we show that a properly trained AI model can mimic medicinal chemists' skills in the autonomous generation of new chemical entities (NCEs) and selection of molecules for experimental validation. This is a prime example of the transfer of the decision power from human experts to AI. Such capabilities could be an important step toward true self-driving laboratories ${ }^{35}$ and serve as an example of the synergy between machine and human intelligence.

In summary, we do not think there is a current universal recipe for optimizing the properties of generated molecules with reinforcement learning. Each task is unique and requires thorough reward function engineering and hyperparameter search. However, as we have demonstrated with the EGFR inhibitor design example, with the right choice of the training protocol, generative models can be a powerful technique for automated and inexpensive de novo molecular design that can be executed even with limited computational and financial resources.

\section{Methods}

In this section, we describe enhancements of deep learning and reinforcement learning approaches used to generate molecules with desired properties. Briefly, we employ the reinforcement learning pipeline introduced in our prior work ${ }^{7}$ with several novel improvements to overcome the problem of sparse rewards. Below we will talk about each part of the pipeline, introduce our novel tricks and heuristics in more detail, and discuss an EGFR case study.

Generative model. For the generative model, we used a deep recurrent neural network with an augmented memory stack described in our previous work. ${ }^{7}$ This network is trained to produce novel molecules in the form of SMILES strings ${ }^{5}$. The network has two modes - training mode and inference mode. In the training mode, the model receives a SMILES string from the training set and tries to reconstruct it, starting from the given prefix. The model is essentially trained as a multiclass classifier, where classes are represented as symbols in the SMILES string alphabet. In the inference mode, instead of receiving prefix from the training set, the model iteratively takes its output as new inputs to generate the next symbol based on the previously generated ones. The generation stops when the network produces a unique stop token interpreted as a command to end generation. The model is implemented as a part of OpenChem ${ }^{36}$ https:/github.com/Mariewelt/OpenChem - an open-source deep-learning toolkit for computational chemistry and drug design.

Reinforcement learning. For the method for shifting the distribution of predicted target activity for generated molecules, we used the policy gradient algorithm ${ }^{37}$. We adapted the problem to a reinforcement learning setting by treating the generative model as the policy network. In this formulation, the generative model predicts the probability of the next action, i.e., adding a new character to the SMILES string prefix. The set of actions is then limited to the SMILES alphabet. The set of states is then limited to all strings in the SMILES alphabet with lengths up to a specific limit $\mathrm{N}$, where $\mathrm{N}$ is a hyperparameter defined by the maximum length of SMILES strings from the 
training dataset. According to the policy gradient algorithm, the objective function to be maximized is defined as the expected reward:

$$
\mathrm{L}(\theta)=-\sum_{i=1}^{N} r\left(s_{N}\right) \cdot \gamma^{i} \cdot \log p\left(s_{i} \mid s_{i-1} ; \theta\right),
$$

where $s_{N}$ is the generated SMILES string, $s_{i}, i=1, \ldots, N$ is the prefix of $s_{N}$ of length $0<i<N$, $\gamma$ is the discount factor, $p\left(s_{i} \mid s_{i-1} ; \theta\right)$ is the transition probability obtained from the generative model, and $r\left(s_{N}\right)$ is the value of the reward function for the generated SMILES string based on the output of the predictive model of EGFR activity.

Exploration and exploitation trade-off. An encounter of a molecule active against a specific target (e.g., EGFR) is a rare event, so the generative model may very infrequently observe promising molecules. Such a scenario will result in over-exploration - a situation when the model mostly experiences low rewards for inactive molecules and receives insufficient signal to shift the distribution of the generated samples. At the same time, our ultimate goal is to generate novel active molecules. Thus, we do not want to over-exploit information about known active molecules from the historical data. We address this problem by complementing the classic policy gradient algorithm with novel heuristics detailed below to balance exploitation and exploration while training the model to maximize the predicted activity of the generated molecules.

(i) Fine-tuning by transfer learning on high-reward examples. The first algorithmic advance we have explored was to fine-tune the model by transfer learning using generated molecules with high rewards as training samples. Fine-tuning means training the model by minimizing crossentropy loss in the same manner as during the pretraining stage. A similar idea has already been introduced in the literature ${ }^{31}$. Our approach differs from previous approaches through our selection process for fine-tuning training samples. We used generated molecules as training samples, whereas the previous work uses historical data with high experimental activities. Overall, finetuning by transfer-learning results in high exploitation and low exploration. With sufficient rounds of fine-tuning, the generative model produces molecules highly similar to those used for finetuning. Thus, training on historical data results in the exploitation of already known chemical scaffolds instead of discovering novel scaffolds. Such an approach could be suitable for the lead optimization process when the goal is to optimize molecules with a prespecified scaffold. In contrast, fine-tuning on generated molecules with high rewards results in the exploitation of scaffolds produced by the generative network and highly scored by the predictive model. Generated scaffolds could be novel, thus increasing their potential in drug discovery applications.

(ii) Experience replay on high-reward molecules. Another technique that we proposed addresses the problem of sparse rewards while maintaining balancing the exploration-exploitation trade-off. To perform experience replay, we save high-reward trajectories (molecules) to the replay buffer. We randomly draw experience samples from the replay buffer during training and let the generative network follow the experience trajectory through teacher forcing ${ }^{38}$. We then calculate the expected reward maximization loss function and apply policy gradient updates to the generative network parameters. The concept of using experience replay for reinforcement learning 
is not new and has previously proven to be an effective training method in the reinforcement learning domain. ${ }^{39-41}$ We propose using this approach to deal with rare high-reward molecules while avoiding over-exploitation. Like the fine-tuning scenario, we utilize generated molecules with high rewards as training examples (or experiences) in the experience replay. Unlike the finetuning scenario, experience replay does not directly enforce specific characters in the generated SMILES string. Instead, it provides feedback in the form of a high reward at the end of the replay episode, resulting in less exploitation.

(iii) Real-time reward shaping. Real-time reward shaping is one more of our proposed advancements to train the neural network more efficiently in a situation when molecules with high rewards are observed rarely. The idea behind this technique is to change the reward function over training dynamically. We shall explain this concept using a threshold reward function and a predictive model returning the active class probability as an illustrative example. A molecule is considered active in these settings if the returned probability exceeds some threshold, such as 0.5 . At the beginning of the training process, very few generated molecules will have such a high probability; instead, there often is a cohort of molecules with probabilities slightly higher than zero. The real-time reward shaping technique helps the model exploit molecules with non-zero predicted probabilities in the absence of good examples. We introduce the probability threshold $p_{0}$ to differentiate between good and bad examples in our threshold reward function:

$$
R(s)=\left\{\begin{array}{c}
r_{\text {pos }}, \text { if } p(s)>p_{0}, \\
r_{\text {neg }}, \text { otherwise, }
\end{array}\right.
$$

where $s$ is the generated molecule, $p(s)$ is the probability of active class returned by the predictive model, $p_{0}$ is the probability threshold, $r_{\text {pos }}$ is the reward value for good examples, and $r_{\text {neg }}$ is the reward value for bad examples. The probability threshold $p_{0}$ is initialized to a small value and dynamically increased during training. After several iterations of training, we generate a large enough batch of molecules with the current model and predict active class probabilities with the predictive model. The threshold $p_{0}$ is increased if the big enough portion of molecules has predicted active class probabilities bigger than the threshold's current value. In our experiments, we started with $p_{0}=0.05$ and increased it by 0.05 when at least $15 \%$ out of 3000 generated molecules have predicted probabilities of active class greater than $p_{0}$.

Case study. The generative model was pretrained using the ChEMBL dataset ${ }^{23}$, which consists of approximately 2 million bioactive molecules. Notably, every molecule from ChEMBL has reported experimental bioactivity for at least one protein target. The pretraining step teaches the generative model to fit the distribution of molecules from the training data. Once pretrained, the generative network is used to sample new molecules from this distribution. Thus, we can assume that pretraining on a dataset of bioactive molecules such as ChEMBL ensures that the generative model will be capable of sampling bioactive-like molecules. This feature is essential to us since our ultimate goal is to produce active molecules to inhibit EGFR.

Activity data and predictive model. The predictive model was trained on historical experimental data of activities for EGFR extracted from ChEMBL. The EGFR training dataset includes bioactivities extracted from ChEMBL 25 (Target ID CHEMBL203). We considered only 
pChEMBL activities with a confidence score of 8 or greater for 'binding' or 'functional' human EGFR assays. Replicate compounds with bioactivity differences larger than one unit on a log scale were excluded. For similar replicate measurements, a single representative assay value was selected for inclusion in the training dataset. Activity values were binarized according to the $1 \mu \mathrm{M}$ cutoff. Chemical data were processed using OpenEye chemistry toolkit. ${ }^{42}$ Standardizer was used for structure canonicalization, JChem 18.2, 2018, ChemAxon (http://www.chemaxon.com). The dataset was curated according to a well-known protocol $^{43}$.

For the predictive model, we used an ensemble of five random forest (RF) classifiers. For features, we used 2,048-bit ECFP fingerprints as implemented in RDKit (https://www.rdkit.org/). We trained five random forest models on a cross-validated dataset to solve a binary classification problem. Each model in the ensemble returns the probability of class "active" for an input molecule. The resulting ensemble prediction is obtained by averaging predictions of all models in the ensemble.

An interesting observation about this dataset is the presence of a privileged scaffold. Around $50 \%$ of active molecules contain quinazoline chemotype $e^{44,45}$, a known hinge binder in kinase inhibitors ${ }^{46}$. From the crystal structures of know EGFR inhibitors, it is known that hydrophobic residues surround quinazoline ring. The aniline group substituted at the 4 position of quinazoline ring and itself quinazoline ring of drugs like gefitinib and erlotinib are bounded by the hydrophobic pocket ${ }^{47,48}$. With such a 4 -anilinoquinazoline prevalence, we expect to see a bias in the predictive model's predictions towards this specific chemotype.

Experimental validation. Compounds that emerged as computational hits were purchased from Enamine (https://www.enaminestore.com/) and resuspended in $100 \%$ DMSO at $10 \mathrm{mM}$ concentration. In vitro experiments were performed at Reaction Biology (https://www.reactionbiology.com/) using a radioactive assay based on the transfer of ${ }^{33} \mathrm{P}$-labelled phosphate from ATP to the kinase substrate ${ }^{49}$. The HotSpot $^{\mathrm{SM}}$ assay utilizes a miniaturized filter binding, where reaction mixtures are spotted onto filter papers. Then reaction mixture binds the radioisotope-labeled catalytic product. Unreacted phosphate is removed via washing of the filter papers. All reactions were carried out at $10 \mu \mathrm{M}$ ATP concentrations.

\section{Acknowledgments}

A.T. acknowledges NIH 1U01CA207160 and ONR N00014-16-1-2311. O.I. acknowledges support from the National Science Foundation (NSF CHE-1802789 and CHE-2041108) and Eshelman Institute for Innovation (EII) award. S.J.C also acknowledges support from the EII. M.K. acknowledges The Molecular Sciences Software Institute (MolSSI) Software Fellowship and NVIDIA Graduate Fellowship. We gratefully acknowledge the support of Jonathan Lefman and hardware donation from NVIDIA Corporation. O.I. also thanks the OpenEye Free Academic Licensing Program for providing a free academic license for their chemistry toolkit.

The SGC is a registered charity (number 1097737) that receives funds from AbbVie, Bayer Pharma AG, Boehringer Ingelheim, Canada Foundation for Innovation, Eshelman Institute for Innovation, Genome Canada, Innovative Medicines Initiative EUbOPEN (No 875510), Janssen, Merck KGaA Darmstadt 
Germany, MSD, Novartis Pharma AG, Ontario Ministry of Economic Development and Innovation, Pfizer, São Paulo Research Foundation-FAPESP, Takeda, and Wellcome.

\section{References}

(1) Sanchez-Lengeling, B.; Aspuru-Guzik, A. Inverse Molecular Design Using Machine Learning: Generative Models for Matter Engineering. Science 2018, 361 (6400), 360-365. https://doi.org/10.1126/science.aat2663.

(2) Schneider, P.; Walters, W. P.; Plowright, A. T.; Sieroka, N.; Listgarten, J.; Goodnow, R. A.; Fisher, J.; Jansen, J. M.; Duca, J. S.; Rush, T. S.; Zentgraf, M.; Hill, J. E.; Krutoholow, E.; Kohler, M.; Blaney, J.; Funatsu, K.; Luebkemann, C.; Schneider, G. Rethinking Drug Design in the Artificial Intelligence Era. Nature Reviews Drug Discovery. 2020. https://doi.org/10.1038/s41573-019-0050-3.

(3) Moret, M.; Friedrich, L.; Grisoni, F.; Merk, D.; Schneider, G. Generative Molecular Design in Low Data Regimes. Nat. Mach. Intell. 2020. https://doi.org/10.1038/s42256020-0160-y.

(4) Jiménez-Luna, J.; Grisoni, F.; Schneider, G. Drug Discovery with Explainable Artificial Intelligence. Nature Machine Intelligence. 2020. https://doi.org/10.1038/s42256-02000236-4.

(5) Weininger, D. SMILES, a Chemical Language and Information System. 1. Introduction to Methodology and Encoding Rules. J. Chem. Inf. Comput. Sci. 1988, 28 (1), 31-36. https://doi.org/10.1021/ci00057a005.

(6) Olivecrona, M.; Blaschke, T.; Engkvist, O.; Chen, H. Molecular De-Novo Design through Deep Reinforcement Learning. J. Cheminform. 2017, 9 (1), 48. https://doi.org/10.1186/s13321-017-0235-x.

(7) Popova, M.; Isayev, O.; Tropsha, A. Deep Reinforcement Learning for de Novo Drug Design. Sci. $A d v$. 2018, 4 (7), eaap7885. https://doi.org/10.1126/sciadv.aap7885.

(8) Segler, M. H. S.; Kogej, T.; Tyrchan, C.; Waller, M. P. Generating Focused Molecule Libraries for Drug Discovery with Recurrent Neural Networks. ACS Cent. Sci. 2018, 4 (1), 120-131. https://doi.org/10.1021/acscentsci.7b00512.

(9) Gómez-Bombarelli, R.; Wei, J. N.; Duvenaud, D.; Hernández-Lobato, J. M.; SánchezLengeling, B.; Sheberla, D.; Aguilera-Iparraguirre, J.; Hirzel, T. D.; Adams, R. P.; Aspuru-Guzik, A. Automatic Chemical Design Using a Data-Driven Continuous Representation of Molecules. ACS Cent. Sci. 2018, 4 (2), 268-276. https://doi.org/10.1021/acscentsci.7b00572.

(10) Popova, M.; Shvets, M.; Oliva, J.; Isayev, O. MolecularRNN: Generating Realistic Molecular Graphs with Optimized Properties. arXiv. 2019.

(11) Jin, W.; Barzilay, R.; Jaakkola, T. Junction Tree Variational Autoencoder for Molecular Graph Generation. arXiv 2018.

(12) Mercado, R.; Rastemo, T.; Lindelöf, E.; Klambauer, G.; Engkvist, O.; Chen, H.; Bjerrum, E. J. Practical Notes on Building Molecular Graph Generative Models. Appl. AI Lett. 2020, ail2.18. https://doi.org/10.1002/ail2.18.

(13) de Cao, N.; Kipf, T. MolGAN: An Implicit Generative Model for Small Molecular Graphs. arXiv. 2018. 
(14) Lim, J.; Hwang, S.-Y.; Moon, S.; Kim, S.; Kim, W. Y. Scaffold-Based Molecular Design with a Graph Generative Model. Chem. Sci. 2020, 11 (4), 1153-1164. https://doi.org/10.1039/C9SC04503A.

(15) Guimaraes, G. L.; Sanchez-Lengeling, B.; Outeiral, C.; Farias, P. L. C.; Aspuru-Guzik, A. Objective-Reinforced Generative Adversarial Networks (ORGAN) for Sequence Generation Models. arXiv 2017.

(16) Putin, E.; Asadulaev, A.; Vanhaelen, Q.; Ivanenkov, Y.; Aladinskaya, A. V.; Aliper, A.; Zhavoronkov, A. Adversarial Threshold Neural Computer for Molecular de Novo Design. Mol. Pharm. 2018, 15 (10), 4386-4397.

https://doi.org/10.1021/acs.molpharmaceut.7b01137.

(17) Blaschke, T.; Engkvist, O.; Bajorath, J.; Chen, H. Memory-Assisted Reinforcement Learning for Diverse Molecular de Novo Design. J. Cheminform. 2020, 12 (1), 68. https://doi.org/10.1186/s13321-020-00473-0.

(18) Jin, W.; Barzilay, R.; Jaakkola, T. Multi-Objective Molecule Generation Using Interpretable Substructures. arXiv $\mathbf{2 0 2 0 .}$

(19) Zhavoronkov, A.; Ivanenkov, Y. A.; Aliper, A.; Veselov, M. S.; Aladinskiy, V. A.; Aladinskaya, A. V.; Terentiev, V. A.; Polykovskiy, D. A.; Kuznetsov, M. D.; Asadulaev, A.; Volkov, Y.; Zholus, A.; Shayakhmetov, R. R.; Zhebrak, A.; Minaeva, L. I.; Zagribelnyy, B. A.; Lee, L. H.; Soll, R.; Madge, D.; Xing, L.; Guo, T.; Aspuru-Guzik, A. Deep Learning Enables Rapid Identification of Potent DDR1 Kinase Inhibitors. Nat. Biotechnol. 2019, 37 (9), 1038-1040. https://doi.org/10.1038/s41587-019-0224-x.

(20) Jin, W.; Barzilay, R.; Jaakkola, T. Junction Tree Variational Autoencoder for Molecular Graph Generation. In 35th International Conference on Machine Learning, ICML 2018; 2018.

(21) Bickerton, G. R.; Paolini, G. V.; Besnard, J.; Muresan, S.; Hopkins, A. L. Quantifying the Chemical Beauty of Drugs. Nat. Chem. 2012. https://doi.org/10.1038/nchem.1243.

(22) Brown, N.; Fiscato, M.; Segler, M. H. S.; Vaucher, A. C. GuacaMol: Benchmarking Models for de Novo Molecular Design. J. Chem. Inf. Model. 2019, 59 (3), 1096-1108. https://doi.org/10.1021/acs.jcim.8b00839.

(23) Mendez, D.; Gaulton, A.; Bento, A. P.; Chambers, J.; De Veij, M.; Félix, E.; Magariños, M. P.; Mosquera, J. F.; Mutowo, P.; Nowotka, M.; Gordillo-Marañón, M.; Hunter, F.; Junco, L.; Mugumbate, G.; Rodriguez-Lopez, M.; Atkinson, F.; Bosc, N.; Radoux, C. J.; Segura-Cabrera, A.; Hersey, A.; Leach, A. R. ChEMBL: Towards Direct Deposition of Bioassay Data. Nucleic Acids Res. 2019. https://doi.org/10.1093/nar/gky1075.

(24) You, J.; Liu, B.; Ying, R.; Pande, V.; Leskovec, J. Graph Convolutional Policy Network for Goal-Directed Molecular Graph Generation. In Advances in Neural Information Processing Systems; 2018.

(25) Cherkasov, A.; Muratov, E. N.; Fourches, D.; Varnek, A.; Baskin, I. I.; Cronin, M.; Dearden, J. C.; Gramatica, P.; Martin, Y. C.; Todeschini, R.; Consonni, V.; Kuz'min, V. E.; Cramer, R. D.; Benigni, R.; Yang, C.; Rathman, J. F.; Terfloth, L.; Gasteiger, J.; Richard, A. M.; Tropsha, A. QSAR Modeling: Where Have You Been? Where Are You Going To? J. Med. Chem. 2014, 57, 4977-5010.

(26) Tropsha, A. Best Practices for QSAR Model Development, Validation, and Exploitation. Mol. Inform. 2010, 29 (6-7), 476-488. https://doi.org/10.1002/minf.201000061.

(27) Thanh-Tung, H.; Tran, T. Catastrophic Forgetting and Mode Collapse in GANs. In Proceedings of the International Joint Conference on Neural Networks; Institute of 
Electrical and Electronics Engineers Inc., 2020.

https://doi.org/10.1109/IJCNN48605.2020.9207181.

(28) Kinase Library - Enamine.

(29) Bemis, G. W.; Murcko, M. A. The Properties of Known Drugs. 1. Molecular Frameworks. J. Med. Chem. 1996, 39 (15), 2887-2893. https://doi.org/10.1021/jm9602928.

(30) Landrum, G. RDKit: Open-source Cheminformatics. https://doi.org/10.2307/3592822.

(31) Merk, D.; Grisoni, F.; Friedrich, L.; Schneider, G. Tuning Artificial Intelligence on the de Novo Design of Natural-Product-Inspired Retinoid X Receptor Modulators. Commun.

Chem. 2018, 1 (1), 1-9. https://doi.org/10.1038/s42004-018-0068-1.

(32) Grygorenko, O. O.; Radchenko, D. S.; Dziuba, I.; Chuprina, A.; Gubina, K. E.; Moroz, Y. S. Generating Multibillion Chemical Space of Readily Accessible Screening Compounds. iScience 2020, 23 (11), 101681. https://doi.org/10.1016/j.isci.2020.101681.

(33) Meggio, F.; Deana, A. D.; Ruzzene, M.; Brunati, A. M.; Cesaro, L.; Guerra, B.; Meyer, T.; Mett, H.; Fabbro, D.; Furet, P.; Dobrowolska, G.; Pinna, L. A. Different Susceptibility of Protein Kinases to Staurosporine Inhibition: Kinetic Studies and Molecular Bases for the Resistance of Protein Kinase CK2. Eur. J. Biochem. 1995, 234 (1), 317-322. https://doi.org/10.1111/j.1432-1033.1995.317 c.x.

(34) Gani, O. A. B. S. M.; Engh, R. A. Protein Kinase Inhibition of Clinically Important Staurosporine Analogues. Nat. Prod. Rep. 2010, 27 (4), 489-498. https://doi.org/10.1039/b923848b.

(35) Häse, F.; Roch, L. M.; Aspuru-Guzik, A. Next-Generation Experimentation with SelfDriving Laboratories. Trends Chem. 2019, 1 (3), 282-291. https://doi.org/10.1016/j.trechm.2019.02.007.

(36) Korshunova, M.; Ginsburg, B.; Tropsha, A.; Isayev, O. OpenChem: A Deep Learning Toolkit for Computational Chemistry and Drug Design. J. Chem. Inf. Model. 2021, acs.jcim.0c00971. https://doi.org/10.1021/acs.jcim.0c00971.

(37) Willia, R. J. Simple Statistical Gradient-Following Algorithms for Connectionist Reinforcement Learning. Mach. Learn. 1992. https://doi.org/10.1023/A:1022672621406.

(38) Williams, R. J.; Zipser, D. A Learning Algorithm for Continually Running Fully Recurrent Neural Networks. Neural Comput. 1989.

https://doi.org/10.1162/neco.1989.1.2.270.

(39) Mnih, V.; Kavukcuoglu, K.; Silver, D.; Rusu, A. A.; Veness, J.; Bellemare, M. G.; Graves, A.; Riedmiller, M.; Fidjeland, A. K.; Ostrovski, G.; Petersen, S.; Beattie, C.; Sadik, A.; Antonoglou, I.; King, H.; Kumaran, D.; Wierstra, D.; Legg, S.; Hassabis, D. Human-Level Control through Deep Reinforcement Learning. Nature 2015. https://doi.org/10.1038/nature14236.

(40) Lillicrap, T. P.; Hunt, J. J.; Pritzel, A.; Heess, N.; Erez, T.; Tassa, Y.; Silver, D.; Wierstra, D. Continuous Control with Deep Reinforcement Learning. In 4th International Conference on Learning Representations, ICLR 2016 - Conference Track Proceedings; 2016.

(41) Tassa, Y.; Doron, Y.; Muldal, A.; Erez, T.; Li, Y.; de Las Casas, D.; Budden, D.; Abdolmaleki, A.; Merel, J.; Lefrancq, A.; Lillicrap, T.; Riedmiller, M. DeepMind Control Suite. arXiv. 2018.

(42) OEChem TK $\mid$ OEChem Toolkit $\mid$ Cheminformatics.

(43) Fourches, D.; Muratov, E.; Tropsha, A. Trust, but Verify: On the Importance of Chemical Structure Curation in Cheminformatics and QSAR Modeling Research. Journal of 
Chemical Information and Modeling. American Chemical Society July 2010, pp 11891204. https://doi.org/10.1021/ci100176x. Quinazoline | C8H6N2 - PubChem.

Bridges, A. J.; Zhou, H.; Cody, D. R.; Rewcastle, G. W.; McMichael, A.; Showalter, H. D. H.; Fry, D. W.; Kraker, A. J.; Denny, W. A. Tyrosine Kinase Inhibitors. 8. An Unusually Steep Structure-Activity Relationship for Analogues of 4-(3-Bromoanilino)6,7-Dimethoxyquinazoline (PD 153035), a Potent Inhibitor of the Epidermal Growth Factor Receptor. J. Med. Chem. 1996, 39 (1), 267-276. https://doi.org/10.1021/jm9503613.

(46) Wells, C. I.; Al-Ali, H.; Andrews, D. M.; Asquith, C. R. M.; Axtman, A. D.; Chung, M.; Dikic, I.; Ebner, D.; Elkins, J. M.; Ettmayer, P.; Fischer, C.; Frederiksen, M.; Gray, N. S.; Hatch, S.; Knapp, S.; Lee, S.; Lücking, U.; Michaelides, M.; Mills, C. E.; Müller, S.; Owen, D.; Picado, A.; Ramadan, K.; Saikatendu, K. S.; Schröder, M.; Stolz, A.; Tellechea, M.; Treiber, D. K.; Turunen, B. J.; Vilar, S.; Wang, J.; Zuercher, W. J.; Willson, T. M.; Drewry, D. H. The Kinase Chemogenomic Set (KCGS): An Open Science Resource for Kinase Vulnerability Identification. bioRxiv. 2019. https://doi.org/10.1101/2019.12.22.886523.

(47) Park, J. H.; Liu, Y.; Lemmon, M. A.; Radhakrishnan, R. Erlotinib Binds Both Inactive and Active Conformations of the EGFR Tyrosine Kinase Domain. Biochem. J. 2012, 448 (3), 417-423. https://doi.org/10.1042/BJ20121513.

(48) Stamos, J.; Sliwkowski, M. X.; Eigenbrot, C. Structure of the Epidermal Growth Factor Receptor Kinase Domain Alone and in Complex with a 4-Anilinoquinazoline Inhibitor. $J$. Biol. Chem. 2002, 277 (48), 46265-46272. https://doi.org/10.1074/jbc.M207135200.

(49) Anastassiadis, T.; Deacon, S. W.; Devarajan, K.; Ma, H.; Peterson, J. R. Comprehensive Assay of Kinase Catalytic Activity Reveals Features of Kinase Inhibitor Selectivity. Nat. Biotechnol. 2011, 29 (11), 1039-1045. https://doi.org/10.1038/nbt.2017. 